\title{
When Betting Odds and Credences Come Apart: More Worries for Dutch Book Arguments
}

\author{
Darren BRADLEY and Hannes LEITGEB
}

If an agent believes that the probability of $E$ being true is $1 / 2$, should she accept a bet on $E$ at even odds or better? Yes, but only given certain conditions. This paper is about what those conditions are. In particular, we think that there is a condition that has been overlooked so far in the literature. We discovered it in response to a paper by Hitchcock (2004) in which he argues for the $1 / 3$ answer to the Sleeping Beauty problem. Hitchcock argues that this credence follows from calculating her fair betting odds, plus the assumption that Sleeping Beauty's credences should track her fair betting odds. We will show that this last assumption is false. Sleeping Beauty's credences should not follow her fair betting odds due to a peculiar feature of her epistemic situation.

\section{Dutch Books}

Suppose that rational agents bet in line with their beliefs. This means that if an agent believes proposition $E$ with certainty, he will bet in favour of the truth of $E$ at any odds, no matter how long. If he believes $E$ with $50 \%$ certainty, he will accept a bet on $E$ that pays twice the stake (or more). If he believes $E$ with $33 \%$ certainty, he will accept a bet on $E$ that pays 3 times the stake (or more). Some writers defined partial beliefs in terms of betting behaviour, making the link constitutive. We have no need for such a strong link. All we need is for there to be a normative link between the belief and the bet. Something like "Other things being equal (risk-neutral, utility linear with money,...), an agent who accepts $E$ with $50 \%$ certainty is rationally permitted to accept a bet on $E$ that pays twice the stake or better". This link is broadly accepted, and will be all we need. The issue that we are interested in within this paper is the "other things".

Assuming agents bet in line with their beliefs, can we say anything about the beliefs an agent may rationally have by looking at the bets they will make? Dutch book arguments say that we can. A Dutch book is a series of bets such that anyone who accepts the bets will end up losing money however the world turns out. A Dutch book argument says that any set of beliefs that justifies an agent's accepting a Dutch book is irrational. The beliefs lead to the bets; the bets leads to a guaranteed loss; therefore the beliefs were irrational. Dutch book arguments have been the main arguments given for probabilism - the doctrine that one's beliefs should conform to the probability calculus (Ramsey 1927; cf. Skyrms 1987). Given the importance of this idea, the argument deserves careful scrutiny. 
A Dutch book argument is also used by Chris Hitchcock (2004); not for probabilism, but in arguing that an agent should have a particular set of beliefs. In the Sleeping Beauty problem (as explained below), the disagreement is about the degree of belief Sleeping Beauty should have that a coin landed Heads. Some argue for $1 / 3$, others for $1 / 2$. Hitchcock points out that $1 / 3$ is the only degree of belief that avoids a Dutch book. We agree with him on this point. Hitchcock concludes that $1 / 3$ is the only rationally permissible belief. We disagree. Various examples have already been given in the literature where correct betting behaviour comes apart from rational degree of belief. Hitchcock is careful to make sure that his example avoids being like any of these cases. But we think he has highlighted a new case, not previously noticed, where betting behaviour should come apart from rational degrees of belief. Thus betting considerations in Sleeping Beauty, as in other cases, are inconclusive.

\section{Sleeping Beauty}

Sleeping Beauty is about to be put to sleep. She will be woken on Monday then put back to sleep. If a fair coin lands Heads, she will not be awoken again. If it lands Tails, she will also be woken on Tuesday. But the drug is such that on Tuesday she will have no memory of the Monday awakening. So she will not know, when awoken, whether it is Monday or Tuesday. And of course this will be true of the Monday awakening as well, as she is not told how the coin lands. When Beauty finds herself awake, what credence should she have in the proposition that the coin landed Heads?

There are two compelling, mutually exclusive arguments.

Half: Her credence should be $1 / 2$, because she has learnt no new evidence that is relevant to the coin landing Heads.

Third: Her credence should be $1 / 3$, because if the experiment is repeated there will be twice as many awakenings due to Tails.

Admittedly this last one is not a very good argument. Hitchcock has a better one. He imagines a bookie who offers Sleeping Beauty various bets, but each on the outcomes of the single coin toss that was described in the story above. The bookie has no more information than Sleeping Beauty (otherwise Dutch books are not a sign of irrationality), so we can imagine him being subject to the same druggings and awakenings as Beauty. Nevertheless, Hitchcock shows that Beauty can avoid being Dutch booked if and only if she assigns Tails a credence of $1 / 3$ on being awoken. Let us review the betting situations that occur in the $1 / 2$ and in the $1 / 3$ case: 
$P(E)=1 / 2$ :

Suppose Beauty refuses to follow Hitchcock's advice, and stubbornly assigns $P(E)=1 / 2$ on being awoken. The bookie then offers the following set of bets: On Sunday, Beauty is offered a bet of $£ 15$ that wins $£ 15$ if Tails lands; on each awakening, Beauty is offered a bet of $£ 10$ that wins $£ 10$ if Heads lands; i.e.:

$\begin{array}{lllll} & \text { Sunday } & \text { Monday } & \text { Tuesday } & \text { Net } \\ \text { Heads } & -15 & 10 & & -5 \\ \text { Tails } & 15 & -10 & -10 & -5\end{array}$

Suppose the coin lands Heads. The first bet loses $£ 15$. The second bet, on Monday, wins $£ 10$. Beauty and the bookie sleep through Tuesday. Overall, Beauty loses $£ 5$. Suppose the coin lands Tails. The first bet wins $£ 15$. The second bet, on Monday, loses $£ 10$. The third bet, on Tuesday, loses $£ 10$. Overall, Beauty loses $£ 5$. Either way, Beauty loses $£ 5$. She has accepted a Dutch book. She did so because she bet in accordance with her $50 \%$ credence that the coin landed Heads.

$P(E)=1 / 3$ :

Disaster can be avoided if Beauty follows Hitchcock's advice: while she first assigns $P(E)=1 / 2$ on Sunday, she changes her assignment to $P(E)=1 / 3$ when awoken. She will not accept the evens bet on Heads when awoken. The deal would have to be sweetened. A layout of $£ 10$ would have to be rewarded with winnings of $£ 20$ (instead of $£ 10$ ), as Heads has fallen in probability:

$\begin{array}{lllll} & \text { Sunday } & \text { Monday } & \text { Tuesday } & \text { Net } \\ \text { Heads } & -15 & 20 & & 5 \\ \text { Tails } & 15 & -10 & -10 & -5\end{array}$

Now the bet looks as it should. She loses $£ 5$ if Tails, and wins $£ 5$ if Heads. It can be shown that no Dutch book can be made against Beauty in this new setting. In order to see this, note that the bookie is not able to distinguish the Monday awakening from the Tuesday awakening himself otherwise he would have more background information than Beauty has, which we want to avoid - so he is not able to come up with two distinct bets on Monday and Tuesday in any systematic manner. Thus, we may assume that he actually offers the same bet twice: now let the money which Beauty would lose on Monday and Tuesday given Tails, respectively, be of amount $x$, and let what she would win on Monday given Heads be of amount $2 x$ or more; if $y$ is what she would 
lose on Sunday given Heads while winning $y$ or more given Tails, then it is impossible that both the total Heads outcome $-y+2 x$ and the total Tails outcome $y-2 x$ are negative, so there is no way Beauty is bound to lose. We can also see that $1 / 3$ is the only probability that leads to "fair bets" on awakening, in the sense that Beauty is equally happy to take either side of the bet. Each waking bet $\operatorname{costs} x$, so $2 x$ is lost if Tails. Whence the fair payoff given Heads must be $2 x$. This bet will be considered fair if and only if Beauty's credence in Heads on waking is 1/3.

Hitchcock concludes that $P(E)=1 / 3$ is the rationally required answer, which tells us Beauty really ought to believe with $1 / 3$ probability that the coin landed Tails. We think this is incorrect. It is true that $1 / 3$ is the only credence that avoids a Dutch book, but we think the example is one in which the agent should not bet in line with her credences. The only way to avoid a Dutch book is to bet as if one believed Heads landed with 1/3 certainty. But from this it does not follow that the agent really is rationally required nor even permitted to believe that Heads landed with $1 / 3$ probability. Let us take a look at a similar example, where betting as if one believed a proposition to $1 / 3$ certainty will avoid a Dutch book.

\section{Separating Credences From Betting Odds}

What we need is a case where it is clear that the probability of a coin landing Heads is $1 / 2$, but nevertheless, one should bet as if the probability was something other than $1 / 2$. This would happen if the bet were only "actually" offered if the coin landed Tails. We propose two ways of getting this result.

\section{Forgery}

Imagine that you knew a fair coin was about to be flipped. If the coin lands Heads, no bet will be made. If the coin lands Tails, you are offered a bet on Heads, but not Heads of a new coin flip but of the flip that has just taken place. Should you accept this bet? Of course not. You should not take a bet, no matter how generous the odds, on the proposition that the coin landed Heads. So perhaps we have a case where your betting odds have come apart from your credences? Not yet; this is no good as it stands, because the fact that you have been offered the bet might tell you that the coin landed Tails. You have received extra information that shifts your credences. So in fact your credence in Tails is close to 1 . And it is therefore in line with your credences not to accept bets on Heads. Credences and betting odds are still aligned.

What we need is a way of making sure that offering the bet does not inform the agent that the coin landed Tails. And we can do that by offering a fake bet. Imagine that instead of no bet being offered if the coin lands Heads, a bet will indeed be offered, except with fake money. Your 
notes, and the bookie's, have been switched for excellent, but worthless, forgeries. Neither you nor the bookie can tell the difference. If the coin lands Tails, you will be offered a bet (on Heads) with real money. If it lands Heads, you will be offered a bet (on Heads) with fake money. Should you take the bet? Of course not. Either the coin lands Tails and you lose real money, or it lands Heads and you win fake money. You are much better off holding onto your real money. Nevertheless, your credence that the coin landed Heads should remain at $1 / 2$. Why should your subjective degree of belief in the outcome of the coin landing event be affected by the existence of a fake bet that you are not even aware of as being fake? So we have a case where your credence that the (fair) coin landed Heads (1/2) should not guide your betting behaviour.

\section{Hallucination}

The point can be made even more vividly by making the example such that the fake bet does not exist at all; it will just be in your head. As in the Sleeping Beauty case, we also add a second time period. Suppose that if the coin lands Tails, you will be offered two real bets on Heads (of the same flip), one after the other. There is no funny business here. But if the coin lands Heads, you will be offered a real bet on Heads and you will also hallucinate being offered a bet on Heads. You won't know whether the hallucination occurs at the first stage or the second stage. You do know that one of the bets will be real and one will be a hallucination. Whether or not you accept the hallucinatory bet, you will later wake up and find your wallet untouched. So we have:

\begin{tabular}{|l|l|l|}
\hline & Stage 1 & Stage 2 \\
\hline Tails & Real Bet & Real Bet \\
\hline Heads & Real Bet or Hallucinatory Bet & $\begin{array}{l}\text { Real if the first bet was } \\
\text { hallucinatory, hallucinatory if } \\
\text { the first bet was real. }\end{array}$ \\
\hline
\end{tabular}

Should you accept any of these bets? No. Your credence in Tails should remain at $50 \%$, but you should not accept either (evens) bet on Heads. Again, we have found a case where your credences and betting odds come apart. Hopefully this is already intuitively correct, but let us go carefully through the reasoning: It is straightforward why the credence should stay the same. You have the same experiences given either Heads or Tails, so you have learnt nothing that could give you relevant information. What about the bets? We can sum over the possible bets to find the expected utility is negative. There are 4 possible bets: 

a) Tails and Real (first bet)
b) Tails and Real (second bet)
c) Heads and Real (first or second bet)
d) Heads and Hallucinatory (first or second bet, in any case not real)

Head and Tails still have a 50\% probability. How do we divide these probabilities up further between a and b (and c and d)? Using Elga's (2004) Restricted Principle of Indifference, accepted by all concerned including Hitchcock (and implicitly used earlier), each of these 4 possibilities gets a probability of $25 \%$. What is the expected value of each one? Let $s$ be the stake. a has a value of $-s$, and $\mathrm{c}$ has a value of $s$ (the bet is even), so these cancel. $\mathrm{d}$ has a value of 0 as no bet is made. The net effect comes from $b$, which has a value of $-s$. So the expected utility is:

$$
1 / 4 *(-s)+1 / 4 *(-s)+1 / 4 * s+1 / 4 * 0=1 / 4 *(-s)
$$

We get negative expected utility, but credence in Tails stays at $50 \%$. If you take a bet with negative expected utility, a Dutch book can be made against you, as shown by Hitchcock. The reason for the negative utility is that one of the bets only exists for real if you are going to lose it. Normally the existence of such an unfair bet would tell the agent what the result is. But the agent's unfortunate epistemic state (hallucinating a bet) prevents him from learning this piece of information. However, this does not stop him from losing money if he takes the bet. So we have a case where credences come apart from betting odds.

\section{Sleeping Beauty Revisited}

It is a small step from this example to Sleeping Beauty. All we need to do is change the hallucination to a state where the agent is not conscious at all; in either case there is no real bet. And to stop Beauty learning from the offer of a second bet, we need to give her amnesia so she does not know whether it is the first or second bet. These are the only two changes needed to turn the example into Sleeping Beauty.

In the Hallucination case, if the first bet was real, then the second bet should not be accepted because it is only offered for real if it is a losing bet. That is, there is only a second real bet if the coin landed Tails, in which case the bet offered on Heads will lose.

In Sleeping Beauty, the Tuesday bet (if there is one) should not be accepted because it is only offered if it is a losing bet. That is, it only exists if the coin landed Tails, so the bet on 
Heads will lose. Beauty does not know if the bet offered to her is the Monday bet (fair) or the Tuesday bet (unfair). So she simply sums the expected utility. She adds the expected utility of the Monday bet (0) to the expected utility of the Tuesday bet (negative). The result is negative. So neither waking bet should be accepted. We can see that the key move in Hitchcock's argument is not the Dutch book as a package, but his claim that the waking bets should be accepted. We have shown that the waking bets should not be accepted as they have negative utility, so the Dutch book is avoided.

In Hallucination, we think the fact that the agent only avoids a Dutch book by betting as if her credence in Heads is $1 / 3$ gives her no reason to actually believe that the probability of Heads is $1 / 3$. Similarly, we think that in Sleeping Beauty the fact that the agent only avoids a Dutch book by betting as if her credence in Heads is $1 / 3$ gives her no reason to actually believe that the probability of Heads is $1 / 3$. Hitchcock must show why the agent's credence in Heads (in Hallucination) should be $1 / 3$, or show that Sleeping Beauty has received some extra piece of information that is omitted from Hallucination. We think neither of these moves are plausible.

What is the reason that in all these cases a subject's credences should differ from his betting rates? Forgery, Hallucination, and Sleeping Beauty have a key feature in common. The trick being played on the agent is that the size or the very existence of a bet on event $E$ is correlated with the outcome of $E$. In Forgery, the agent is offered a large bet if they are betting on the wrong result, i.e., on what has not happened, and a small bet (of value zero) if they are betting on the correct result. In Hallucination, we also get a correlation between the size of a bet and the outcome of the corresponding event. This is because one of the bets is only actually made if the agent is betting on the wrong result - another way of getting a bet of size zero in the winning situation. There are various other examples in the literature of cases where an agent should not make bets in accordance with her credences. One example is Talbott's (1991) where the agent has less information than the bookie. Further ones are Maher's (1993) and Seidenfeld et al. (1990) where utilities are not linear in money. Another is that the agent is irrational at a future time (Christensen 1991). Hitchcock avoids these problems, but we think he has not noticed the one we point out - where the size of a bet on an event is correlated with the outcome of the event. It is not surprising this has not been noticed before. It is a very peculiar epistemic state to be in, such that you are only offered a bet given a certain condition, but nevertheless the offer of the bet does not tell you that the condition is satisfied.

Hitchcock has attempted to give a Dutch book argument to the conclusion that only particular credences are rationally acceptable. This result assumes that if only particular betting 
odds will avoid a Dutch book, these odds should guide our credences. We have attempted to show that Sleeping Beauty is (yet another) case where credence and betting odds diverge. If either the size or the existence of a bet on $E$ is correlated with the result of $E$ and the agent is at the same time unaware of the size or existence of the bet, a Dutch book can be constructed. But the Dutch book argument does not help us figure out what Sleeping Beauty should believe. If there is a normative link between beliefs and bets, then the corresponding norm should include the proviso: "Other things being equal (risk-neutral, utility linear with money,..., and: it is not that case that the agent is unaware of the fact that the size or the existence of the bet is correlated with the outcome of the event that the bet is on)...".

Stanford University Stanford, CA 94305-2155, USA dbradley@stanford.edu University of Bristol 9 Woodland Rd., Clifton, Bristol, UK BS8 1TB Hannes.Leitgeb@bristol.ac.uk

\section{BIBLIOGRAPHY}

Christensen, D. 1991. Clever Bookies and Coherent Beliefs. Philosophical Review 100: 229-47. Elga, A. 2004. Defeating Dr. Evil with Self-Locating Belief. Philosophy and Phenomenological Research 69(2).

Hitchcock, C. 2004. Beauty and the Bets. Synthese 139(3): 405-20.

Maher, P. 1993. Betting on Theories. Cambridge: Cambridge University Press.

Ramsey 1927. Truth and Probability. In Philosophical Papers, ed. D. H. Mellor. Cambridge:

Cambridge University Press.

Seidenfeld, T. 1990. When Fair Betting Odds Are Not Degrees of Belief. PSA vol.1: 517-524.

Skyrms, B. 1987. Coherence. In Scientific Inquiry in Philosophical Perspective, ed. N. Rescher.

Pittsburgh, PA: University of Pittsburgh Press.

Talbott, W. 1991. Two Principle of Bayesian Epistemology. Philosophical Studies 62: 135-50.

\footnotetext{
${ }^{1}$ We want to thank Christopher Hitchcock for various very helpful discussions on a preliminary draft of the paper.
} 